\title{
BAYESIAN ESTIMATION IN AN IMAGE RESTORATION PROBLEM IN X-RAY FIBER DIFFRACTION
}

\author{
Shyamsunder Baskaran and R. P. Millane \\ Computational Science and Engineering Program, \\ Whistler Center for Carbohydrate Research, \\ Purdue University, West Lafayette, IN 47907
}

\begin{abstract}
The restoration of an incomplete image from a known part and experimental data in the form of the Fourier amplitude squared sums is formulated as a Bayesian estimation problem. This problem is motivated by the structure completion problem in $\mathrm{x}$-ray fiber diffraction analysis. An appropriate prior of uniformly distributed impulses is used. The Bayesian MMSE and MAP estimates are obtained. Simulations are used to compare the performance of the estimates. The results show that the MMSE estimate significantly outperforms the other estimates. The restored images exhibit some bias towards the known part of the image. This can be partly reduced by an unbiasing procedure.
\end{abstract}

\section{INTRODUCTION}

$\mathrm{X}$-ray crystallography is used to study three dimensional (3-D) molecular structures at atomic resolution. A crystalline specimen of the molecule under study is irradiated by a monochromatic $\mathrm{x}$-ray beam, and the resultant scattering pattern (diffraction pattern) is recorded for a variety of specimen orientations. Since the scattering is weak, the diffracted wave field is the Fourier transform of the electron density distribution of the molecule. A crystalline (periodic) specimen, which is required to obtain a usable signal to noise ratio, results in the diffraction pattern being a Nyquist sampled Fourier transform of one period of the image. The measured data (intensity) are the squares of these samples. The support of one period of the image is termed the unit cell.

The image $\varrho(\mathbf{x})$ is represented as the convolution of the electron density of one period $\rho(\mathbf{x})$ with the crys- tal lattice $l(\mathbf{x})$, i.e. ,

$$
\varrho(\mathbf{x})=\rho(\mathbf{x}) \otimes l(\mathbf{x}),
$$

where $l(\mathbf{x})=\sum_{(m, n, p) \in \mathbb{Z}^{3}} \delta(\mathbf{x}-m \mathbf{a}-n \mathbf{b}-p \mathbf{c})$. The Fourier Transform (FT) of $\varrho(\mathbf{x})$ is

$$
F_{\mathbf{h}}=F(\mathbf{u}) L(\mathbf{u})
$$

where $F(\mathbf{u})=\mathcal{F}[\rho(\mathbf{x})], \mathcal{F}$ is the Fourier transform, $\mathbf{h}=(h, k, l), L(\mathbf{u})=\mathscr{F}[l(\mathbf{x})]$ which is also a lattice $\sum_{\mathbf{h} \in \mathbb{Z}^{3}} \delta\left(\mathbf{u}-h \mathbf{a}^{*}-k \mathbf{b}^{*}-l \mathbf{c}^{*}\right)$ and $F_{\mathbf{h}}=F(\mathbf{u}=$ $\left.h \mathbf{a}^{*}-k \mathbf{b}^{*}-l \mathbf{c}^{*}\right) . L(\mathbf{u})$ is called the reciprocal lattice.

$\mathrm{X}$-ray fiber diffraction is a crystallographic technique used to study polymers that do not form regular crystals [1]. The specimen is called a fiber and is composed of an aggregate of small crystallites that are randomly rotated about a preferred axis. This results in cylindrical averaging of the intensities in Fourier space. Owing to the symmetry of the reciprocal lattice $L(\mathbf{u})$, the observations are then of the form

$$
I_{j}=\sum_{\mathbf{h} \in \mathcal{S}_{j}}\left|F_{\mathbf{h}}\right|^{2},
$$

where $\delta_{j}$ is the set of reciprocal lattice points of the same cylindrical polar radius.

An important and practical problem that occurs in fiber diffraction (XFD) involves completing the image function $\rho(\mathbf{x})$, from the intensity data, and a partial image $\rho^{P}(\mathbf{x})$. This occurs in structural biology where the 3-D structure (image) may consist of known (located in 3-D) components and other unknown components (such as other molecules, ions or solvent molecules) that need to be located [1]. 
Labeling the missing contribution to the image as $\rho^{Q}(\mathbf{x})$, we have

$$
\rho(\mathbf{x})=\rho^{P}(\mathbf{x})+\rho^{Q}(\mathbf{x}),
$$

so that

$$
F_{\mathrm{h}}=F_{\mathrm{h}}^{P}+F_{\mathrm{h}}^{Q}
$$

and the problem reduces to one of estimating $\rho^{Q}(\mathbf{x})$, or equivalently $F_{\mathrm{h}}^{Q}$, from $I_{j}$ and $F_{\mathrm{h}}^{P}$.

\section{STATISTICAL ESTIMATION}

We adopt the following notation. Each observation $I_{j}$ represents information from $n_{j}=2\left|\mathcal{S}_{j}\right|$ components, the real and imaginary parts of each contributing $F_{\mathbf{h}}$, $\mathbf{h} \in S_{j}$. Denoting these $n_{j}$ components as vectors, defined as

$\mathbf{Y}=\left[\begin{array}{c}A_{h_{1}} \\ B_{h_{1}} \\ \vdots \\ B_{h_{n / 2}}\end{array}\right] ; \mathbf{X}=\left[\begin{array}{c}A_{h_{1}}^{Q} \\ B_{h_{1}}^{Q} \\ \vdots \\ B_{h_{n / 2}}^{Q}\end{array}\right] ; \boldsymbol{\Theta}=\left[\begin{array}{c}A_{h_{1}}^{P} \\ B_{h_{1}}^{P} \\ \vdots \\ B_{h_{n / 2}}^{P}\end{array}\right] ;$

so that for each $j, \mathbf{Y}=\mathbf{X}+\Theta$ ( $j$ is suppressed), and $I_{j}=\|\mathbf{Y}\|^{2}$.

We apply a prior model to obtain the density function for the $X_{i}$ based on the fact that the structure consists of atoms, i. e. sharp separated peaks of electron density. The image is therefore considered to be composed of impulses, belonging to sets denoted by $\mathcal{N}, \mathcal{P}$ and $Q$ for the complete, known and missing parts, respectively. The number of impulses in each of these sets are $|\mathcal{N}|,|\mathcal{P}|$ and $|\mathcal{Q}|$. Taking the members of the set $Q$ to be independent and uniformly distributed in the unit cell, the components of $\mathbf{X}$ are independent and identically distributed, $N\left(0, \Sigma_{Q} / 2\right)$, where $N(a, b)$ is a normal pdf with mean $a$ and variance $b$, and $\Theta$ is deterministic (known). Applying Bayes' rule, we obtain the conditional density, $P_{\mathbf{X} \mid I_{j}}(\cdot)$, the posterior density for the missing structure given the observations, as

$$
\begin{aligned}
P_{\mathbf{X} \mid I_{j}}(\mathbf{x})= & \left(\pi \Sigma_{Q}\right)^{n_{j} / 2} \\
& \exp \left(-\|\mathbf{X}\|^{2} / \Sigma_{Q}\right) \delta\left(I_{j}-\|\mathbf{x}+\boldsymbol{\Theta}\|^{2}\right) .
\end{aligned}
$$

We use this conditional density to estimate $F_{\mathbf{h}}^{Q}$. In current XFD analysis, heuristic estimates are used to estimate the Fourier coefficients and hence the missing image. We use the posterior to obtain the minimum mean square error estimate, and have also shown the current heuristic's to correspond to certain MAP estimates [2].

The minimum mean square error (MMSE) estimate is the conditional mean of the posterior density, i. e.

$$
\hat{\mathbf{X}}^{\mathrm{MMSE}}=\int_{\mathbf{x}} \mathbf{x} P_{\mathbf{X} \mid I_{j}}(\mathbf{x}) d \mathbf{x}
$$

which can be evaluated to give [2]

$$
\hat{F}_{\mathbf{h}}^{Q \text { MMSE }}=\left(\frac{I_{n_{j} / 2}(\chi)}{I_{n_{j} / 2-1}(\chi)} \sqrt{\frac{I_{j}}{I_{j}^{P}}}-1\right) F_{\mathbf{h}}^{P},
$$

$\forall \mathbf{h} \in \mathcal{S}_{j}$, where $I_{\nu}(\cdot)$ is the modified Bessel function of the first kind of order $\nu$ and $\chi=2\left(I_{j} I_{j}^{P}\right)^{1 / 2} / \Sigma_{Q}$.

The maximum a posteriori (MAP) estimate given by

$$
\hat{\mathbf{X}}^{\mathrm{MAP1}}=\arg \max _{\mathbf{x}} P_{\mathbf{X} \mid I_{j}}(\mathbf{x}),
$$

can be evaluated to gives the Fourier coefficients [2]

$$
\hat{F}_{\mathbf{h}}^{Q M A P 1}=\left(\sqrt{\frac{I_{j}}{I_{j}^{P}}}-1\right) F_{\mathbf{h}}^{P} .
$$

The complex Fourier coefficients $F_{\mathrm{h}}^{P}$ may be expressed in modulus - phase angle form as $\left|F_{\mathbf{h}}^{P}\right| \exp \left(i \phi_{\mathbf{h}}^{P}\right)$. Using only the information from the phase angle (to reduce the bias towards $\rho^{P}(\mathbf{x})$, we may obtain another MAP estimate given by

$$
\hat{\mathbf{X}}^{\mathrm{MAP2}}=\arg \max _{\mathbf{x}} P_{\mathbf{X} \mid I_{j},\left\{\phi_{\mathbf{h}}^{P} \mid \mathbf{h} \in \mathrm{S}_{j}\right\}}(\mathbf{x}),
$$

which give the Fourier coefficients [2]

$$
\hat{F}_{\mathbf{h}}^{Q \mathrm{MAP2}}=\left(\sqrt{\frac{I_{j}}{n_{j}}}-1\right) F_{\mathbf{h}}^{P} .
$$

The MAP1 and MAP2 estimates correspond to current practice in XFD analysis [1].

\section{THEORY OF BIAS EFFECTS}

An estimate $\hat{G}$ of a random variable $G$ is said to be biased when the expected values $(\langle\cdot\rangle$ denotes expectation) differ, i. e.

$$
\langle\hat{G}\rangle \neq\langle G\rangle
$$


When the bias in the estimation is towards some known parameter, say $T$, the estimate $\hat{G}$, may be broken down into components that are parallel and orthogonal to $T$, so that the orthogonal component which will be less biased may be used.

The bias in the estimation of the Fourier coefficients arise because the posterior density function is centered about $\Theta$. While the MMSE estimates are unbiased in general, the same cannot be said of the MAP estimates for which $\left\langle\hat{F}_{\mathbf{h}}^{Q}\right\rangle \neq\left\langle F_{\mathbf{h}}^{Q}\right\rangle$. We may "unbias" these estimates by removing the component of $\hat{\rho}^{Q}(\mathbf{x})$ that is correlated with $\rho^{P}(\mathbf{x})$. This "decorrelated" estimate is a better estimate of the missing part of the structure, and is calculated as

$$
\hat{\rho}^{U}(\mathbf{x})=\hat{\rho}^{Q}(\mathbf{x})-B^{\prime} \rho^{P}(\mathbf{x}),
$$

where $B^{\prime}=B\left[\left(\int \hat{\rho}^{Q}(\mathbf{x})^{2} d \mathbf{x}\right)\right]^{1 / 2}$, and $B$ is the bias coefficient defined in the next section.

\section{SIMULATIONS}

In order to obtain performance metrics on the estimates, simulations were performed on random sets of 2-D images. The images consist of uniformly distributed unit impulses. The fraction of the image that is missing is quantified using $\Delta Q=|\mathcal{Q}| /|\mathcal{N}|$. Fourier amplitudes were randomly combined into an equal number of sets $\delta_{j}$, such that $1 \leq\left|\mathcal{S}_{j}\right| \leq S_{\max }$ to obtain the data $I_{j}$. The data loss is quantified as $S_{\max }$. The quality of the reconstruction for the different estimates was measured using the correlation coefficient of the estimated image $\hat{\rho}^{Q}(\mathbf{x})$ with $\rho^{Q}(\mathbf{x})$, i. e.

$$
C=\frac{\int \hat{\rho}^{Q}(\mathbf{x}) \rho^{Q}(\mathbf{x}) d \mathbf{x}}{\left[\left(\int \rho^{Q}(\mathbf{x})^{2} d \mathbf{x}\right)\left(\int \hat{\rho}^{Q}(\mathbf{x})^{2} d \mathbf{x}\right)\right]^{1 / 2}} .
$$

Since the partial structure information forms a bias for estimating the missing density, the estimates $\hat{\rho}^{Q}(\mathbf{x})$ tend to be correlated with the known partial structure $\rho^{P}(\mathrm{x})$. The bias is quantified using

$$
B=\frac{\int \hat{\rho}^{Q}(\mathbf{x}) \rho^{P}(\mathbf{x}) d \mathbf{x}}{\left[\left(\int \rho^{P}(\mathbf{x})^{2} d \mathbf{x}\right)\left(\int \hat{\rho}^{Q}(\mathbf{x})^{2} d \mathbf{x}\right)\right]^{1 / 2}} .
$$

\section{RESULTS AND DISCUSSION}

$32 \times 32$ pixel images were used in the simulations. We chose $|\mathcal{N}|=50$ and $|Q| \in\{5,10\}$ so that $\Delta Q$ was 0.10

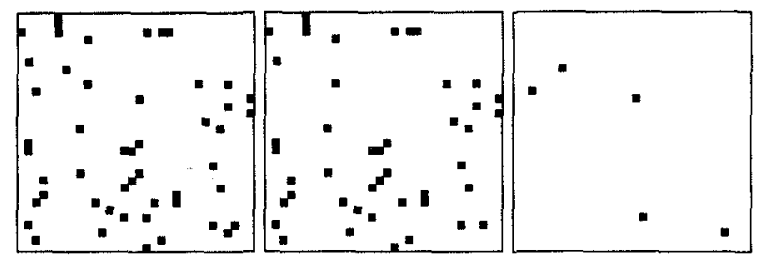

Figure 1: Test images used for the simulations. Left to Right: $\rho(\mathbf{x}), \rho^{P}(\mathbf{x})$ and $\rho^{Q}(\mathbf{x})$ for $\Delta Q=0.1$.

and 0.20. These are shown in (Fig. 1). For each set $S_{\max }$ was varied from 1 to 5 , and the MMSE, MAP1 and MAP2 estimates of $\hat{\rho}^{Q}(\mathbf{x})$ were computed. The correlation and bias coefficients were then evaluated.

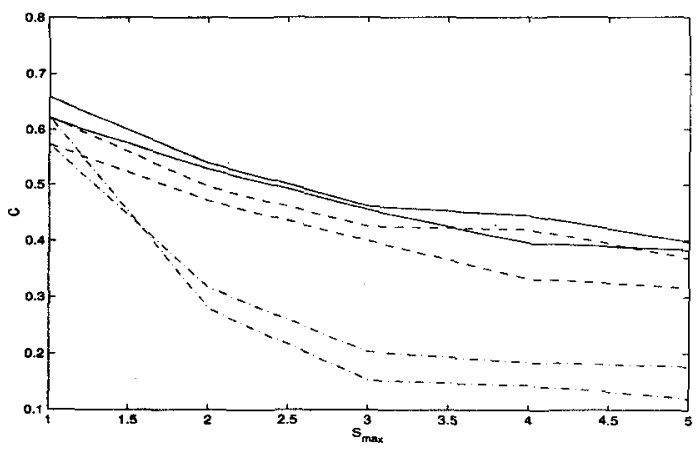

Figure 2: Correlation in the MMSE (-), MAP1 (--) and MAP2 (-..-) estimates for $\Delta Q=0.10$ (upper curves) and 0.20 (lower curves).

The correlation coefficients (Fig. 2) decrease with both $\Delta Q$ and $S_{\max }$. The MMSE estimates perform better than MAP1 and MAP2, especially for large amounts of missing image and data loss. Fig. 3 shows reconstructed images for $\Delta Q=0.1$. While the MAP estimates compare well with the MMSE at low $\Delta Q$ and $S_{\max }$, the MMSE estimate is superior at higher values.

The bias towards $\rho^{P}(\mathbf{x})$ increases (Fig. 4) with increasing $S_{\max }$, and $\Delta Q$ in general. An exception is the MAP2 estimate whose bias decreases with $S_{\max }$. However, the low correlation coefficients for this estimate indicates poor quality, and the absence of bias is of no real consequence. The MMSE coefficients have an $I_{n_{j} / 2}(\chi) / I_{n_{j} / 2-1}(\chi)$ "weight" which reflects the uncertainty associated with using the Fourier coefficients of the known part to break down (or phase) the intensity datum. This produces the least biased of the estimates. 

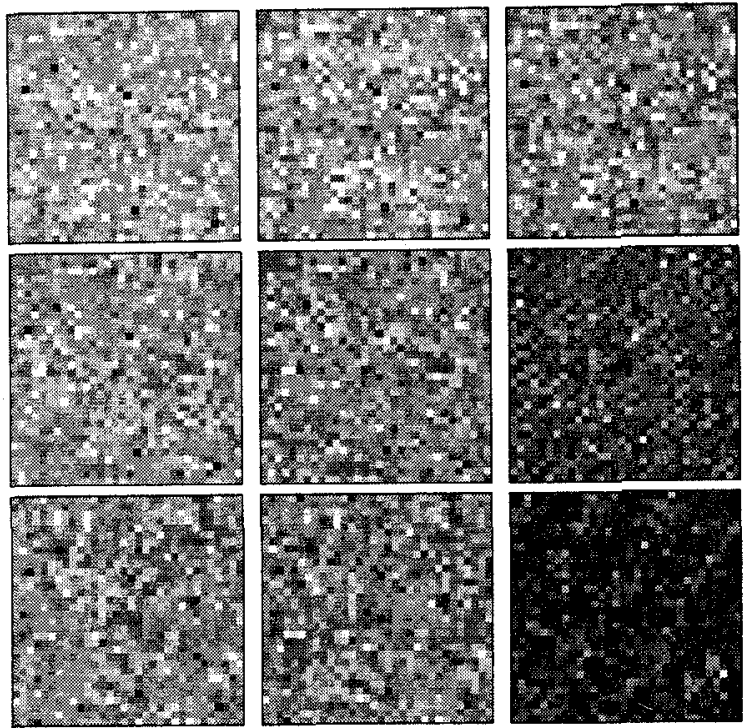

Figure 3: Estimated images $\hat{\rho}^{Q}(\mathbf{x})$ from the simulations with $\Delta Q=0.1$. Left to Right: MMSE, MAP1 and MAP2 reconstructions, Top to Bottom: $S_{\max }=1,2,3$

The images are decorrelated in real space by removing the component that is parallel to the known image using (15). The performance curves before and after the decorrelation operation are shown for $\Delta Q=0.4$ in Fig. 5. The MAP1 estimate shows some improvement, while the MAP2 estimate shows marginal improvement. The MMSE shows no improvement indicating its unbiased nature and still possesses higher correlation with the true image than do the decorrelated estimates. Images for the MAP1 estimate for $\Delta Q=0.2$ and $S_{\max }=$ 2, with and without decorrelation are shown in Fig. 6. A minor improvement is seen in the decorrelated estimate, mainly as reduced background noise.

Acknowledgements: Supported by NSF (DBI-9722862).

\section{REFERENCES}

[1] R. P. Millane, "Structure determination by $x$-ray fiber diffraction," In Crystallographic computing 4: Techniques and new Technologies, Isaacs, N. W. and Taylor, M. R. Eds., Oxford Univ. Press, pp. 169-186, 1988.

[2] S. Baskaran and R. P. Millane, "Bayesian image reconstruction in x-ray fiber diffraction involving partial image and spectral amplitude data," IEEE Trans. Image Process., Submitted, 1997.

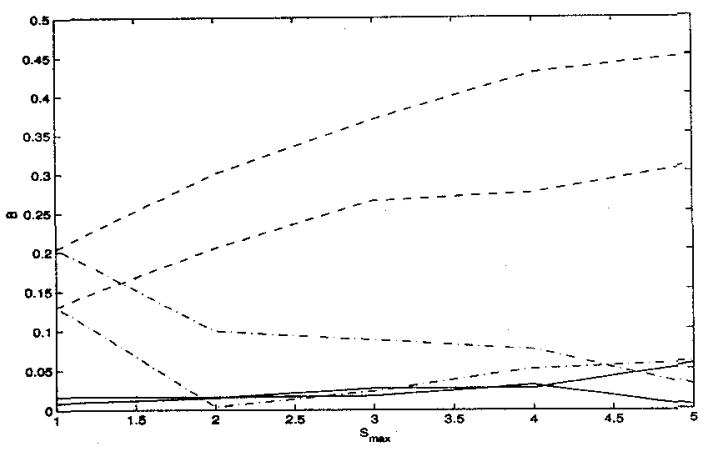

Figure 4: Bias in the MMSE (-), MAP1 (-) and MAP2 (-.-.) estimates for $\Delta Q=0.10$ (lower curves) and 0.20 (upper curves).

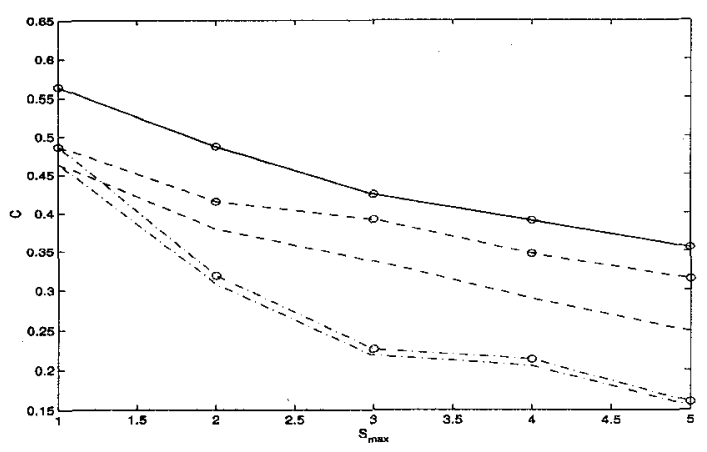

Figure 5: Correlation in the MMSE ( $\longrightarrow$, MAP1 (-- ) and MAP2 (-..-) estimates for $\Delta Q=0.40$, before (lines) and after (with circles) decorrelation.

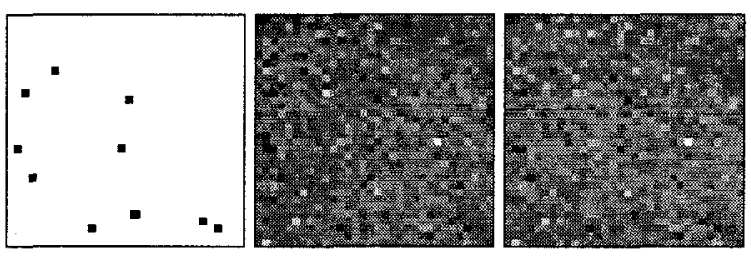

Figure 6: True image (left) for $\Delta Q=0.2$ and $S_{\max }=$ 2, and MAP1 (center) and decorrelated MAP1 (right) estimates. 